\title{
Association between periodontal disease and Vitamin D status in a type 1 diabetic population
}

Ramos L, Casanova L, Piedra M, Muñoz P, García Unzueta MT, Amado JA. Sección de endocrinología. Hospital Universitario Marqués de Valdecilla. Cantabria.

\section{Background and aims}

Periodontal disease (PD) is a chronic inflammatory condition where the key feature is the alveolar bone loss. Diabetes Mellitus is a major risk factor in the occurrence of PD and Vitamin D has been found to play a role in the development of diseases such as PD for its immunomoduratory and anti-inflammatory effects. The purpose of this study was to evaluate the relationship between PD and vitamin D levels in type 1 diabetic patients.

\section{Subjects and Methods}

We conducted a prospective longitudinal survey since December 2010 to December 2011. Type 1 diabetic patients aged between 18 and 65 years were recruited. Their periodontal status were examined, metabolic parameters were registered and $25 \mathrm{OH}(\mathrm{D}), 1,25 \mathrm{OH}(\mathrm{D})$ and $\mathrm{PTH}$ were measured.

\section{Results}

\begin{tabular}{|c|c|c|}
\hline & & Type 1 DM \\
\hline & $n$ & Mean \pm SD \\
\hline Age (yr) & 59 & $44 \pm 11,6$ \\
Years of DM evolution(yr) & 58 & $19 \pm 9,6$ \\
Sex (\%) & 59 & $56 \%$ males \\
& & $44 \%$ females \\
BMl (kg/m2) & 59 & $26 \pm 4,3$ \\
HbA1c (\%) & 59 & $7,8 \pm 1,09$ \\
Calcium p (mg/dl) & 48 & $9,3 \pm 0,3$ \\
Albumin (g/dl) & 48 & $4,2 \pm 0,2$ \\
Calcium ion (mmol/l) & 48 & $1,23 \pm 0,04$ \\
PTH (pg/ml) & 49 & 50 \\
25 (OH) D (ng/ml) & 49 & $26,5 \pm 11,8$ \\
1,25 (OH) D (pg/ml) & 49 & $53,1 \pm 18,4$ \\
Osteocalcin (ng/ml) & 49 & 11 \\
& & \\
\hline
\end{tabular}
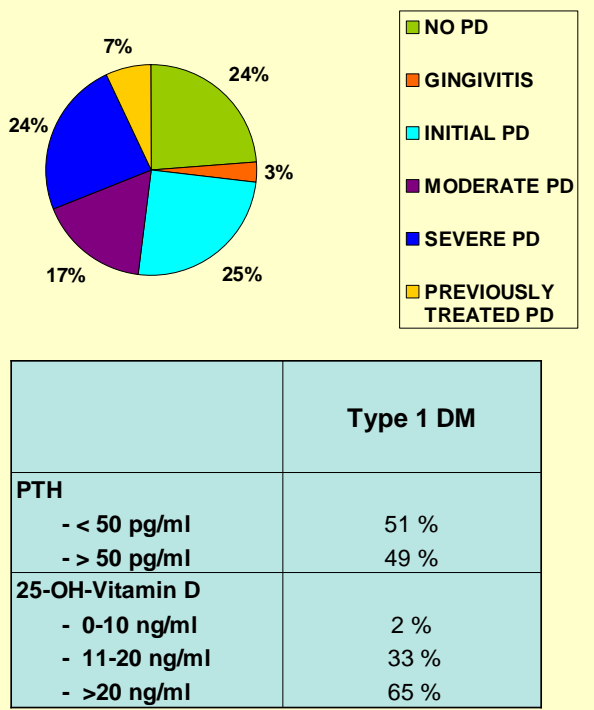

PD was significantly associated with age and systolic blood pressure. Levels of 25(OH)D were inversely associated with PTH $(r=-0,521 ; p \varangle 0.01)$ and directly associated with $1,25(\mathrm{OH}) \mathrm{D}(\mathrm{r}=0,369, \mathrm{p}<0,05)$. In regression analysis the only variable that showed to be significantly associated with the degree of PD was 1,25(OH)D, being high levels protectors of PD (OR: 0,953 , IC: $0,910-0,998)$. This means that a decrease of $10 \mathrm{pg} / \mathrm{ml}$ increases the risk of PD at 2.69 compared to individuals with $1,25(\mathrm{OH}) \mathrm{D}$ normal levels.

\section{Conclusions}

1. We found in this study high prevalence of PD in type 1 diabetic patients. Only $24 \%$ of patients do not have any degree of PD.

2. Low serum 1,25 $(\mathrm{OH}) \mathrm{D}$ concentrations may be associated with increased PD severity.

3. Future studies are needed to prospectively assess the beneficial effect of vitamin D of periodontal disease. 